\title{
Staged endovascular treatment of a coexisting parasellar aneurysm and endoscopic resection of a pituitary macroadenoma: illustrative case
}

\author{
Aishwarya Nene, MPhil, ${ }^{1}$ Christopher S. Hong, MD, ${ }^{2}$ Declan McGuone, MBBCh, ${ }^{3}$ Charles C. Matouk, MD, ${ }^{2}$ and S. Bulent Omay, MD \\ ${ }^{1}$ Yale School of Medicine and Departments of ${ }^{2}$ Neurosurgery and ${ }^{3}$ Pathology, Yale School of Medicine, New Haven, Connecticut
}

BACKGROUND Intracranial aneurysms and pituitary adenomas are relatively common pathologies that, in rare instances, may concurrently present. Their management poses considerable clinical and technical challenges.

OBSERVATIONS The authors present a case of a 66-year-old female patient with a fusiform aneurysm of the left internal carotid artery associated with a symptomatic pituitary macroadenoma that had been causing visual deficits for the past several years. She underwent successful placement of flow diverter stents across her aneurysm, followed by routine dual antiplatelet therapy to maintain stent patency. She underwent frequent serial radiographic, endocrine, and ophthalmological evaluations during this time to ensure stability of her pituitary adenoma. Following confirmation of aneurysm obliteration and subsequent de-escalation of antiplatelet medications to aspirin monotherapy, her tumor was subsequently resected via an endoscopic endonasal approach in a delayed fashion.

LESSONS The authors review the literature regarding management of these concurrent pathologies and describe the aspects of the case that led them to their chosen treatment strategy. An algorithm is proposed regarding the management of parasellar aneurysms with a concurrent diagnosis of pituitary tumor pathology.

https://thejns.org/doi/abs/10.3171/CASE21699

KEYWORDS intracranial aneurysm; pituitary adenoma; endonasal endoscopic approach; fusiform aneurysm; parasellar

Aneurysms associated with pituitary tumors have been well documented in the literature, and they have an incidence of approximately $3.6 \%$, based on several series. ${ }^{1}$ The management of these pathologies is clinically challenging, particularly in cases in which patients are symptomatic from their pituitary tumors and are also at high risk for spontaneous or iatrogenic rupture of their aneurysm. We describe surgical management of a patient with a symptomatic pituitary macroadenoma with an incidentally diagnosed large, fusiform parasellar aneurysm herniating into the tumor.

\section{Illustrative Case}

A 66-year-old female presented with several years of progressive visual decline, prompting further work-up with magnetic resonance imaging $(\mathrm{MRI})$ that showed a $2.8 \times 2.1 \times 3.8-\mathrm{cm}$ suprasellar mass compressing the optic chiasm, consistent with a pituitary macroadenoma (Fig. $1 \mathrm{~A}$ and $\mathrm{B}$ ). Furthermore, there was noted fusiform dilation of the left internal carotid artery (ICA) in the paraophthalmic region. Computed tomography angiography (CTA) revealed a fusiform aneurysm measuring $9 \mathrm{~mm}$ in greatest diameter and notably herniating into the tumor (Fig. $1 \mathrm{C}$ and D). The patient's neurological examination was notable for bitemporal hemianopsia with 20/70 right eye (OD) and 20/60 left eye (OS) visual acuity. There were no biochemical endocrine abnormalities other than mild central hypothyroidism, characterized by low free thyroxine $(0.71$ $\mathrm{ng} / \mathrm{dL}$; reference range, $0.8-1.8 \mathrm{ng} / \mathrm{dL}$ ) and normal thyroid-stimulating hormone (TSH) $(2.630 \mu \mathrm{lU} / \mathrm{mL}$; reference range, $0.300-4.200$ $\mu \mathrm{IU} / \mathrm{mL}$ ), for which she was started on levothyroxine therapy.

Given her visual deficits, surgical intervention was recommended but required addressing the aneurysm first, given the risk of rupturing the left ICA aneurysm herniated into the tumor during an

ABBREVIATIONS CTA = computed tomography angiography; EEA = endoscopic endonasal approach; ICA = internal carotid artery; MRI = magnetic resonance imaging; $\mathrm{OD}=$ right eye; $\mathrm{OS}=$ left eye; $\mathrm{TSH}=$ thyroid-stimulating hormone.

INCLUDE WHEN CITING Published March 7, 2022; DOI: 10.3171/CASE21699.

SUBMITTED December 12, 2021. ACCEPTED January 20, 2022.

(C) 2022 The authors, CC BY-NC-ND 4.0 (http://creativecommons.org/licenses/by-nc-nd/4.0/). 

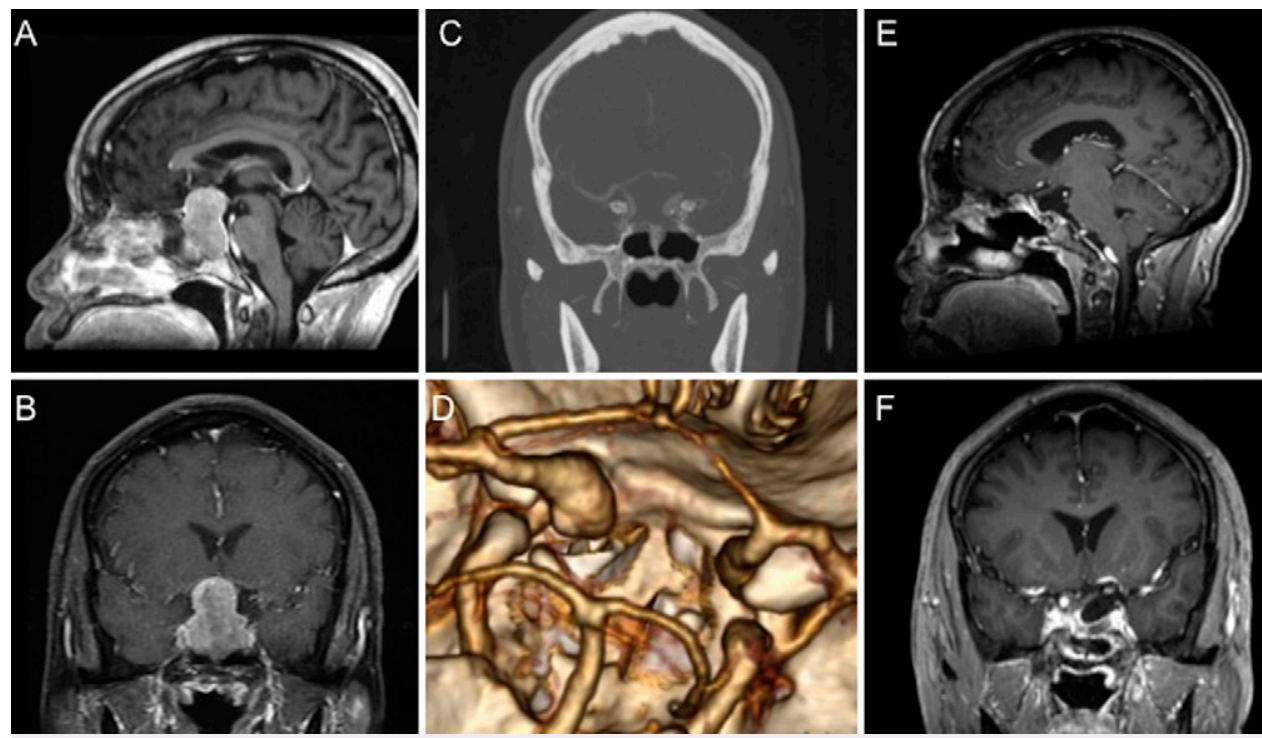

FIG. 1. Preoperative imaging. A large, 3.8-cm enhancing sellar lesion is seen on sagittal (A) and coronal (B) T1-weighted postcontrast MRI, consistent with a pituitary macroadenoma. A 7-mm medially directed left parasellar ICA aneurysm is shown on a coronal image (C) from a bone-windowed CTA and a three-dimensional reconstruction (D) centered on the sella. Repeat imaging obtained 7 months after pituitary adenoma surgery shows no evidence of tumor recurrence on sagittal $(\mathbf{E})$ and coronal $(\mathbf{F})$ T1-weighted postcontrast MRI.

endoscopic endonasal approach (EEA). Considering the fusiform nature of the aneurysm, endovascular options were either treatment with overlapping flow diversion stents or carotid sacrifice. Subsequently, the patient underwent a temporary balloon occlusion test but did not tolerate it. At this point, endovascular stenting was recommended, which would require delaying tumor surgery at least 6 months while she received antiplatelet therapy. The patient was counseled on the risk of further visual deterioration in the interim and the potential for spontaneous intratumoral hemorrhage during this time. However, given the relatively long-standing nature of her visual decline, this treatment strategy was believed to be the safest option.

The patient underwent uncomplicated endovascular placement of three overlapping stents (Fig. 2A-C) and was continued on aspirin and clopidogrel therapies. Serial neuro-ophthalmological studies obtained at 1,6 , and 12 months post-procedurally revealed stable visual deficits. Repeat biochemical testing 2 and 6 months postoperatively did not reveal any new endocrine abnormalities. Surveillance MRI scans obtained 1 and 12 months post-procedurally showed stable size of the tumor. Six months after endovascular treatment, nearly complete aneurysmal obliteration was confirmed on digital subtraction angiography (Fig. 2D-F), and the patient was continued on aspirin monotherapy with discontinuation of clopidogrel. A follow-up CTA 6 months later showed complete obliteration of the aneurysm with a widely patent stent construct, at which point she was cleared for tumor surgery with temporary cessation of aspirin in the perioperative period. She subsequently underwent endoscopic endonasal resection of her sellar tumor with intraoperative use of vascular Doppler to localize the ICAs. The finding of final pathology was a nonfunctional pituitary adenoma with a Ki-67 index $<3 \%$ (Fig. 3). Neuro-ophthalmological examination 2 months postoperatively revealed significant improvement of her bitemporal hemianopsia and 20/30 OD, 20/40 OS visual acuity. Follow-up MRI scans obtained 3 and 7 months after surgery showed stable postoperative changes without evidence of recurrent tumor (Fig. $1 \mathrm{E}$ and F).

\section{Discussion}

\section{Observations}

The management of coexistent pituitary tumors and parasellar aneurysms is varied and highly tailored to patient-specific pathology. Raper et al. ${ }^{2}$ reported the largest case series of management of sellar pathologies and parasellar aneurysms in 13 patients, which included elective staged coiling or carotid sacrifice before EEA for pituitary tumor resection, as well as stent-assisted coiling but in the setting of a ruptured aneurysm shortly after resection of a pituitary adenoma, highlighting the potential morbidity of upfront endoscopic endonasal surgery before securing the aneurysm. In cases of truly intrasellar aneurysms arising from the cavernous or supraclinoid portion of the ICA and eroding into the sella, aneurysm treatment before pituitary surgery has been performed for obvious reasons of aneurysmal rupture during sellar removal of pituitary tumors. ${ }^{2-5}$ In our patient, we did not visualize the aneurysm directly during surgery, because it remained on the other side of the sellar diaphragm. However, one case from the Raper et al. series demonstrated rupture of a previously undetected suprasellar aneurysm projecting inferomedially into a pituitary macroadenoma within hours after uncomplicated gross total resection of the pituitary tumor. ${ }^{2}$ In the absence of the pituitary tumor, our practice would have been to observe the aneurysm for growth, based on data on the natural history of unruptured aneurysms. ${ }^{6,7}$ Taking all of this information together, we thought that induced traction on nearby structures during tumor removal would have likely posed excessive risk for aneurysmal rupture, necessitating aneurysm treatment before pituitary surgery.

Given the fusiform nature of our patient's aneurysm, endovascular options were limited to flow diversion stenting or parent vessel sacrifice or an open extracranial-intracranial bypass procedure. Bypass procedures have long been a viable treatment for large fusiform aneurysms, but their use has diminished with the advent of flow diverter endovascular technologies. Bypass procedures have been described to treat fusiform aneurysms of the ICA in 

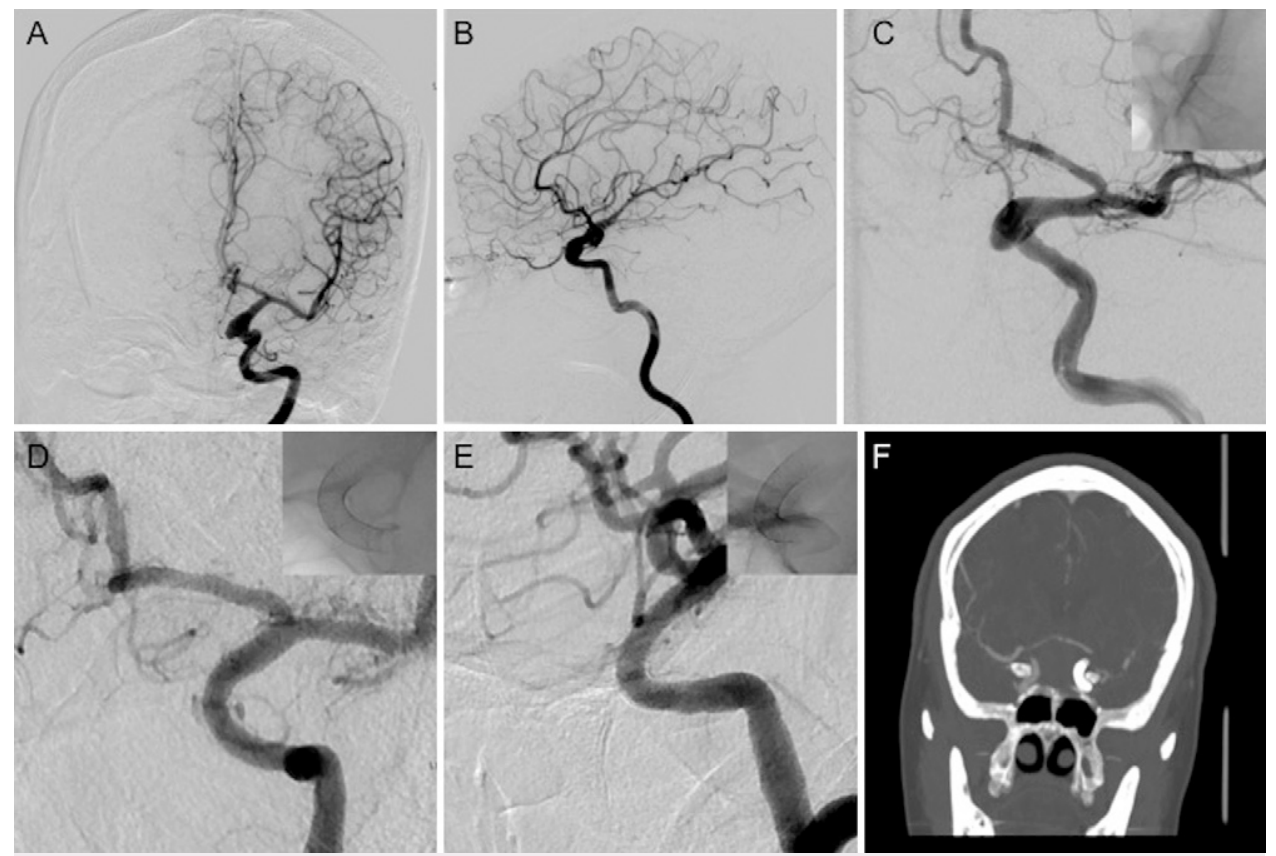

FIG. 2. Digital subtraction angiography. Anterior-posterior (A) and lateral (B) views of a left ICA injection show a medially directed parasellar fusiform aneurysm. C: A lateral oblique view is shown after stent deployment (inset) across the aneurysm. Postoperative anterior-posterior $(\mathbf{D})$ and lateral $(\mathbf{E})$ views of a selective left ICA injection obtained 6 months after stenting shows near obliteration of the aneurysm with a patent stent construct (insets). F: A coronal view of a bone-windowed CTA obtained 1 year after stenting shows complete obliteration of the aneurysm.

association with previously resected or recurrent craniopharyngiomas, but they have typically been pursued after failure of first-line

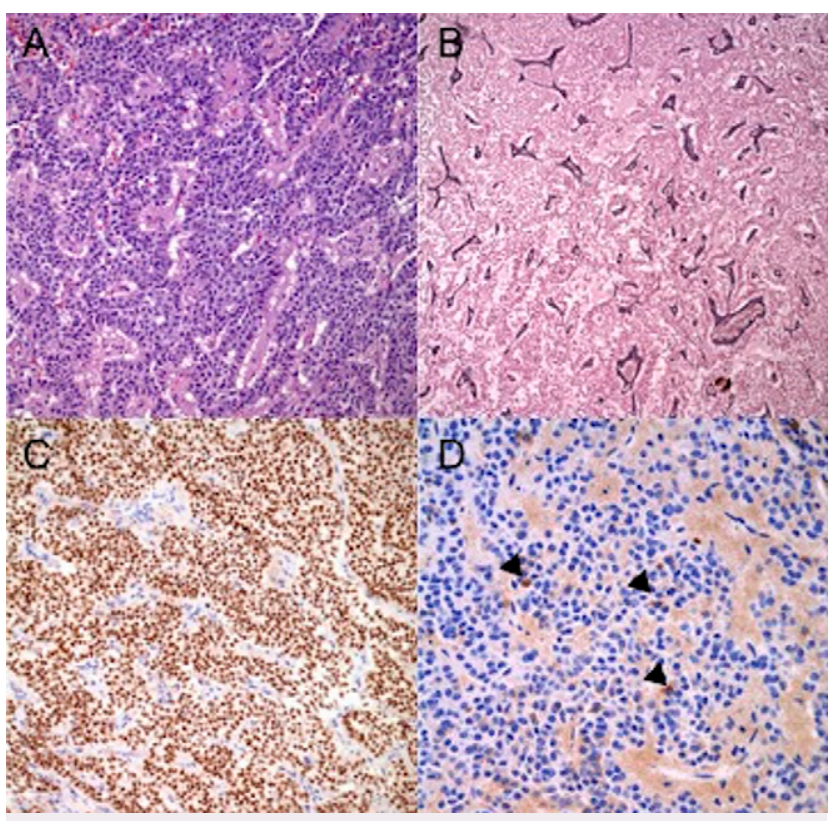

FIG. 3. Histopathology of the pituitary adenoma. A representative hematoxylin and eosin stain (A) is shown with effacement of reticulin (B). The tumor exhibits diffuse neuronal positivity for transcription factor SF1 (C) and contains rare follicle-stimulating hormone-positive cells shown with black arrows (D). In all images, original magnification is $200 x$. endovascular treatment. ${ }^{8}$ In our patient, we chose endovascular intervention with flow diverter stents as the least invasive treatment after confirming that parent vessel takedown was not an option. This strategy exposed the possibility for further visual decline from tumor growth, but, given our patient's long-standing visual deficits over several years, the risk of upfront tumor resection seemed to be outweighed by the possibility of clinical decline from postponed surgery, with only $5 \%-10 \%$ of nonfunctioning pituitary adenomas demonstrating aggressive behavior with rapid growth, invasiveness, and early recurrence. ${ }^{9}$ Previous studies have also shown the postoperative visual outcome after surgery for pituitary adenomas was good in the long run, regardless of the duration of the symptoms. $^{10,11}$

We performed frequent serial endocrine, ophthalmological, and radiographic evaluations of our patient in the postoperative period after aneurysm treatment to monitor for signs of tumor progression, which should be considered paramount in this unique situation. We also considered the risk of spontaneous hemorrhage while our patient was receiving dual antiplatelet therapy, particularly given that prior studies have shown that larger tumor size and use of antithrombotic therapies were significantly associated with risk of hemorrhagic pituitary apoplexy. ${ }^{12,13}$ In contrast, a prior case series showed that EEA for sellar tumor removal while patients were receiving antithrombotic therapy did not differ in terms of intraoperative bleeding or operation time compared with patients not receiving antithrombotic medication, ${ }^{14}$ raising the possibility that EEA for pituitary adenoma resection could be performed soon after an endovascular procedure to treat a parasellar aneurysm in a patient receiving antiplatelet therapy. Given our patient's clinical stability leading up to her tumor diagnosis, we chose to pursue endovascular stenting with 
the understanding that spontaneous hemorrhagic apoplexy while receiving dual antiplatelet therapy may necessitate urgent surgical intervention. We routinely perform delayed digital subtraction angiography 6-12 months after flow diversion stenting and will de-escalate dual antiplatelet therapy to aspirin monotherapy upon confirmation of aneurysm obliteration and stent patency. The patient is then maintained on aspirin monotherapy for life. During this time period, aspirin monotherapy can safely be discontinued for dental/medical procedures such as teeth cleaning, tooth extraction, colonoscopy, or elective surgery and resumed thereafter. As such, we anticipated tumor resection could occur within this time frame after determining our patient no longer required dual antiplatelet therapy and with temporary discontinuation of aspirin in the perioperative period. Had there been visual deterioration or apoplexy before aneurysm obliteration and stent endothelialization, we would have proceeded to reverse the antiplatelet agents immediately before surgery and would have resumed them as soon as possible, likely the day after surgery. Further studies are needed to better quantify the risk of symptomatic intratumoral hemorrhage of pituitary adenomas while a patient receives antiplatelet or anticoagulant medications.

\section{Lessons}

This case highlights that in carefully selected patients, a staged approach to treat a concurrent ICA aneurysm and sellar tumor may be successful. A proposed framework for the management of these coexisting pathologies is illustrated in Fig. 4. Although the data are limited, there is still no clear evidence to suggest that the risk of spontaneous pituitary hemorrhagic apoplexy is higher while patients

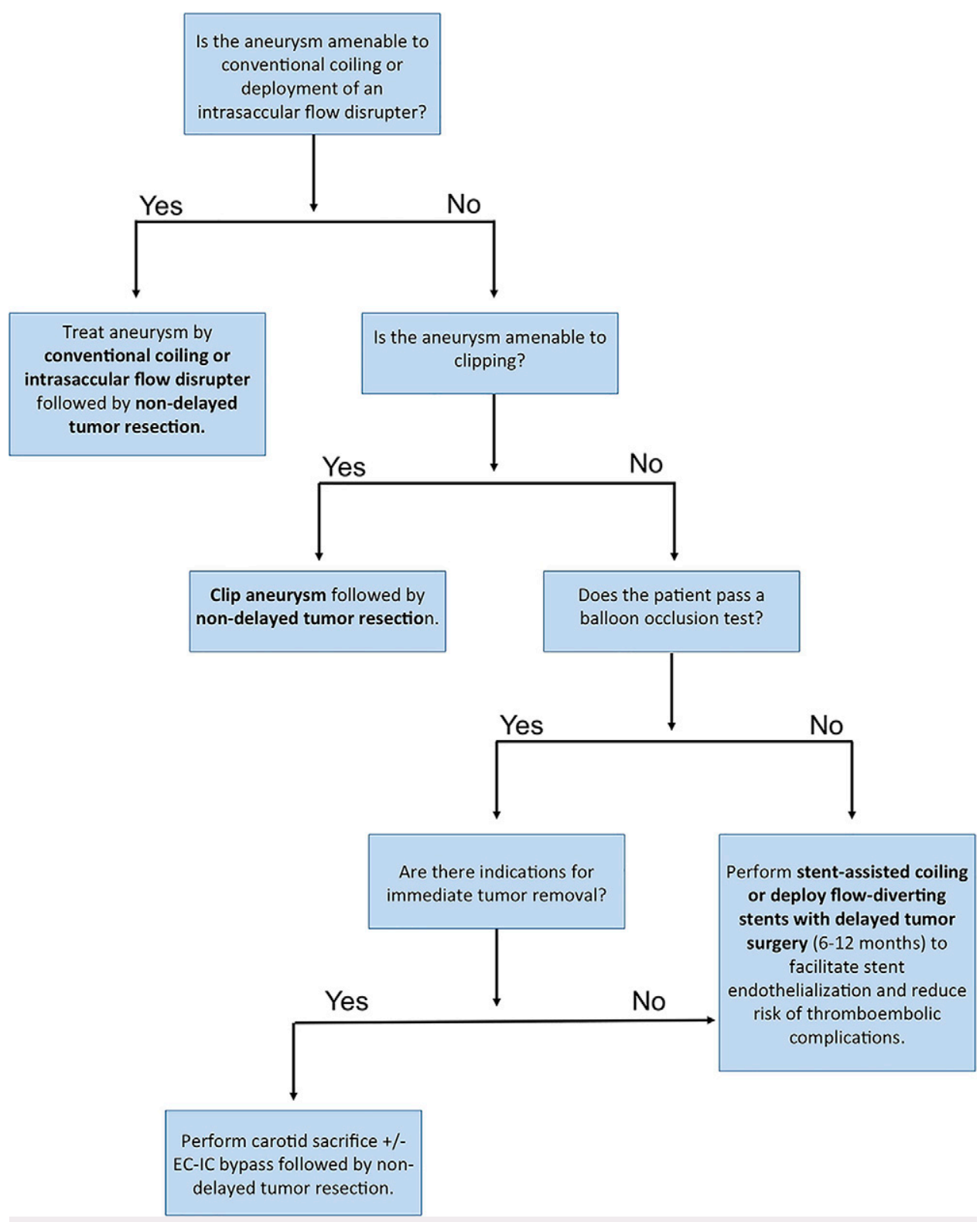

FIG. 4. A proposed framework for treatment of patients with pituitary tumors with coexisting parasellar aneurysms. EC-IC = extracranial-intracranial. 
are receiving antiplatelet therapies. Serial radiographic, endocrine, and ophthalmological evaluations are crucial to monitor for signs of pituitary tumor progression in the period after aneurysm treatment that may necessitate earlier intervention.

\section{References}

1. Bulsara KR, Karavadia SS, Powers CJ, Paullus WC. Association between pituitary adenomas and intracranial aneurysms: an illustrative case and review of the literature. Neurol India. 2007;55(4): 410-412.

2. Raper DM, Ding D, Evans E, et al. Clinical features, management considerations and outcomes in case series of patients with parasellar intracranial aneurysms undergoing anterior skull base surgery. World Neurosurg. 2017;99:424-432.

3. Sade B, Mohr G, Tampieri D, Rizzo A. Intrasellar aneurysm and a growth hormone-secreting pituitary macroadenoma. Case report. J Neurosurg. 2004;100(3):557-559.

4. Seda L Jr, Cukiert A, Nogueira KC, Huayllas MK, Liberman B. Intrasellar internal carotid aneurysm coexisting with $\mathrm{GH}$-secreting pituitary adenoma in an acromegalic patient. Arq Neuropsiquiatr. 2008:66(1):99-100.

5. Yang MY, Chen C, Shen CC. Cavernous aneurysm and pituitary adenoma: management of dual intrasellar lesions. J Clin Neurosci. 2005;12(4):477-481.

6. Morita A, Kirino T, Hashi K, et al. The natural course of unruptured cerebral aneurysms in a Japanese cohort. $N$ Engl J Med. 2012;366(26):2474-2482.

7. Wiebers DO, Whisnant JP, Huston J 3rd, et al. Unruptured intracranial aneurysms: natural history, clinical outcome, and risks of surgical and endovascular treatment. Lancet. 2003;362(9378):103-110.

8. Wang L, Shi X, Liu F, Qian H. Bypass surgery to treat symptomatic fusiform dilation of the internal carotid artery following craniopharyngioma resection: report of 2 cases. Neurosurg Focus. 2016;41(6):E17.

9. Yavropoulou MP, Tsoli M, Barkas K, Kaltsas G, Grossman A. The natural history and treatment of non-functioning pituitary adenomas (non-functioning PitNETs). Endocr Relat Cancer. 2020;27(10): R375-R390.
10. Omay SB, Schwartz TH. Visual outcome after pituitary adenoma surgery. Neurol India. 2016;64(6):1254-1255.

11. Dutta P, Gyurmey T, Bansal R, et al. Visual outcome in 2000 eyes following microscopic transsphenoidal surgery for pituitary adenomas: protracted blindness should not be a deterrent. Neurol India. 2016;64(6):1247-1253.

12. Möller-Goede DL, Brändle M, Landau K, Bernays RL, Schmid C. Pituitary apoplexy: re-evaluation of risk factors for bleeding into pituitary adenomas and impact on outcome. Eur $\mathrm{J}$ Endocrinol. 2011;164(1):37-43

13. Briet C, Salenave S, Bonneville JF, Laws ER, Chanson P. Pituitary apoplexy. Endocr Rev. 2015;36(6):622-645.

14. Ogawa Y, Tominaga T. Sellar and parasellar tumor removal without discontinuing antithrombotic therapy. J Neurosurg. 2015;123(3): 794-798.

\section{Disclosures}

The authors report no conflict of interest concerning the materials or methods used in this study or the findings specified in this paper.

\section{Author Contributions}

Conception and design: Omay, Nene, Hong. Acquisition of data: Omay, Nene, Hong. Analysis and interpretation of data: Omay, Nene, Hong, McGuone. Drafting the article: Nene, Hong. Critically revising the article: Omay, Nene, Hong, Matouk. Reviewed submitted version of manuscript: all authors. Administrative/technical/material support: Omay. Study supervision: Omay, Hong.

\section{Supplemental Information}

Previous Presentations

This research was presented as an e-poster at the AANS Annual Scientific Meeting, August 21-25, 2021, Miami, FL.

\section{Correspondence}

S. Bulent Omay: Yale School of Medicine, New Haven, CT. sacit. omay@yale.edu. 